\title{
A MODIFIED CHI-SQUARED GOODNESS-OF-FIT TEST FOR THE KUMARASWAMY GENERALIZED INVERSE WEIBULL DISTRIBUTION AND ITS APPLICATIONS
}

\section{HAFIDA GOUAL and NACIRA SEDDIK-AMEUR}

Laboratory of Probability and Statistics

University of Badji Mokhtar

Annaba

Algeria

e-mail: hafida-06@hotmail.fr

naciraseddik@yahoo.fr

\begin{abstract}
In this paper, we have proposed and study a new five parameter generalized inverse Weibull model that is based on the cumulative distribution function of Kumaraswamy [14] distribution. The importance of this model lies in its ability to model a monotone and non-monotone failure rate functions, which are quite common to lifetime data analysis and reliability. We present a new goodness-of-fit test proposed by Bagdonavicius and Nikulin [2] for the Kumaraswamy generalized inverse Weibull model in the case of censored data. The method of maximum likelihood is used to estimate the model parameters. We illustrate the model and the proposed test by applications to two real data sets.
\end{abstract}

2010 Mathematics Subject Classification: 60E15, 62E10, 62F12, 62G10, 62 N05.

Keywords and phrases: censored data, generalized inverse Weibull distribution, information matrix, Kumaraswamy distribution, maximum likelihood estimation, Nikulin Rao Robson statistic.

Received December 7, 2016

(C) 2016 Scientific Advances Publishers 


\section{Introduction}

In the survival and reliability analysis, the observed data are frequently incomplete (censoring times). Therefore, it's necessary to consider a test of the censored failure times data. In 2011, Bagdonavicius and Nikulin developed the idea of Akritas [1] of comparing observed and expected numbers of failures in time intervals. The choice of random grouping intervals used are considered as data functions, introducing a modified chi-squared test $Y_{n}^{2}$ which is well adapted to censored failure time data. To give more objective assessments of the selected model of fit, the $Y_{n}^{2}$ statistic is based on the maximum likelihood estimation (MLE), and the Fisher matrix which measure the information about the parameters contained in the model chosen. In the recent years, several criterion statistics and their applications are considered, such as Haghighi and Nikulin [23], Gerville-Réache et al. [17], Voinov et al. [32], Bagdonavicius et al. [4], Nikulin and Tran [24], Goual and Seddik-Ameur [9].

We introduce in this paper, the generalized inverse Weibull (GIW) model based on the cumulative distribution function of Kumaraswamy [14] and called Kumaraswamy generalized inverse Weibull (Kum-GIW) distribution. Till now, the Kumaraswamy distribution has been finding a different areas of applications, such as survival analysis, reliability, biological and hydrological data, Saulo et al. [29], Pascoa et al. [26], Cordeiro et al. [5], Nadarajah et al. [18], Cordeiro et al. [6], Shahbaz et al. [30].

On the other hand, the generalized inverse Weibull (GIW) distribution proposed by de Gusmão et al. [7] is a flexible model which can describe and predict the failure times of much real systems. The importance of the new Kum-GIW distribution lies in its ability to model monotonicity, non-monotonicity, and $\cap$-shape failure rate functions, which are quite common to lifetime data analysis and reliability. The 
purpose of this paper is to construct and analyze the generalized NikulinRao-Robson goodness-of-fit statistic test $Y_{n}^{2}$ (Bagdonavicius and Nikulin [2], Bagdonavicius and Nikulin [3]) for the Kumaraswamy generalized inverse Weibull (Kum-GIW) distribution in both of complete and censored data. We introduce the Kum-GIW distribution and we discuss the shapes of the hazard rate function in Section 2. In Section 3, we calculate the maximum likelihood estimators of the Kum-GIW parameters in the case of censored data. We define in Section 4, the new goodness-of-fit statistic test $Y_{n}^{2}$, and the validation of our new model is investigated in Section 5. Finally, the importance of the proposed model is illustrated by two real data sets in Section 6.

\section{Kumaraswamy Generalized Inverse Weibull Distribution}

The generalized inverse Weibull (GIW) distribution is proposed by Gusmão et al. [7]. This distribution arises as a tractable lifetime model in actuarial sciences, life testing and reliability.

The random variable $T$ follows the generalized inverse Weibull distribution if its cumulative distribution function is given by

$$
F_{G I W}(t, \theta)=\exp \left\{-\gamma\left(\frac{\alpha}{t}\right)^{\beta}\right\}, \theta=(\alpha, \beta, \gamma)^{T}>0, t>0 .
$$

Its probability density function is

$$
f_{G I W}(t, \theta)=\gamma \beta \alpha^{\beta} t^{-(\beta+1)} \exp \left\{-\gamma\left(\frac{\alpha}{t}\right)^{\beta}\right\}, t>0, \theta>0 .
$$

We propose in this paper, an extension of the generalized inverse Weibull distribution based on the family of generalized Kumaraswamy distributions (denoted Kum-G), introduced by Cordeiro and de Castro [6] and Nadarajah et al. [18]. The Kumaraswamy (Kum) distribution is not very common to statisticians and has been little explored in the 
statistical literature. Its cumulative distribution function is $F(x)=1-\left(1-x^{a}\right)^{b}$, for $0<x<1$ and where $a>0$ and $b>0$ are shape parameters, and its density function has a simple form $f(x)=a b x^{a-1}\left(1-x^{a}\right)^{b-1}$.

A random variable $T$ follows a Kumaraswamy generalized inverse Weibull (Kum-GIW) distribution, if the cumulative distribution function is given as

$$
F(t, \theta)=1-\left[1-\exp \left\{-a \gamma\left(\frac{\alpha}{t}\right)^{\beta}\right\}\right]^{b}, t>0, \theta=(a, b, \alpha, \beta, \gamma)^{T}>0,
$$

where $a>0, b>0, \beta>0$ are the shape parameters, $\alpha>0$ is the scale parameter, and $\gamma>0$ is the shift parameter.

Its survival function is

$$
S(t, \theta)=\left[1-\exp \left\{-a \gamma\left(\frac{\alpha}{t}\right)^{\beta}\right\}\right]^{b}, t>0, \theta=(a, b, \alpha, \beta, \gamma)^{T}>0 .
$$

The probability density function, hazard and cumulative hazard functions of the Kum-GIW distribution are

$$
\begin{aligned}
& f(t, \theta)=a b \gamma \beta \alpha^{\beta} t^{-(\beta+1)} \exp \left\{-a \gamma\left(\frac{\alpha}{t}\right)^{\beta}\right\}\left[1-\exp \left\{-a \gamma\left(\frac{\alpha}{t}\right)^{\beta}\right\}\right]^{b-1}, \\
& \theta=(a, b, \alpha, \beta, \gamma)^{T}>0
\end{aligned}
$$

$\lambda(t, \theta)=\frac{f(t, \theta)}{S(t, \theta)}$

$$
\begin{aligned}
&=a b \gamma \beta \alpha^{\beta} t^{-(\beta+1)} \exp \left\{-a \gamma\left(\frac{\alpha}{t}\right)^{\beta}\right\}\left[1-\exp \left\{-a \gamma\left(\frac{\alpha}{t}\right)^{\beta}\right\}\right]^{-1}, t>0, \theta>0, \\
& \Lambda(t, \theta)=-\ln \left(1-\exp \left\{-a \gamma\left(\frac{\alpha}{t}\right)^{\beta}\right\}\right)^{b}, t>0, \theta=(a, b, \alpha, \beta, \gamma)^{T}>0,
\end{aligned}
$$

respectively. 
Where, the cumulative distribution function of a generalized Kumaraswamy distribution is $F_{K u m}(t, \theta)=1-\left[1-\{G(t)\}^{a}\right]^{b},(G(t)$ is the cumulative distribution function of the generalized inverse Weibull distribution).

The graphs of hazard rate functions are shown below (Figure 1) for various choices of the parameters.

The flexibility of the Kum-GIW distributions is shown in their hazard function plots (Figure 1) with interesting properties:

- If $0<\beta \leq 2$ and $1<\gamma<4$, then its hazard rate has a cup shape (П-shape) form.

- If $\beta>2.1$ and $0<\gamma \leq 1$, then its hazard rate is decreasing to 0 , which can characterize the systems that improves (burn-in phase or youth, "burn in" infant mortality).

- If $\gamma \geq 4$, then the hazard rate is increasing, this situation characterizes a system which deteriorates (phase obsolescence or ageing). 

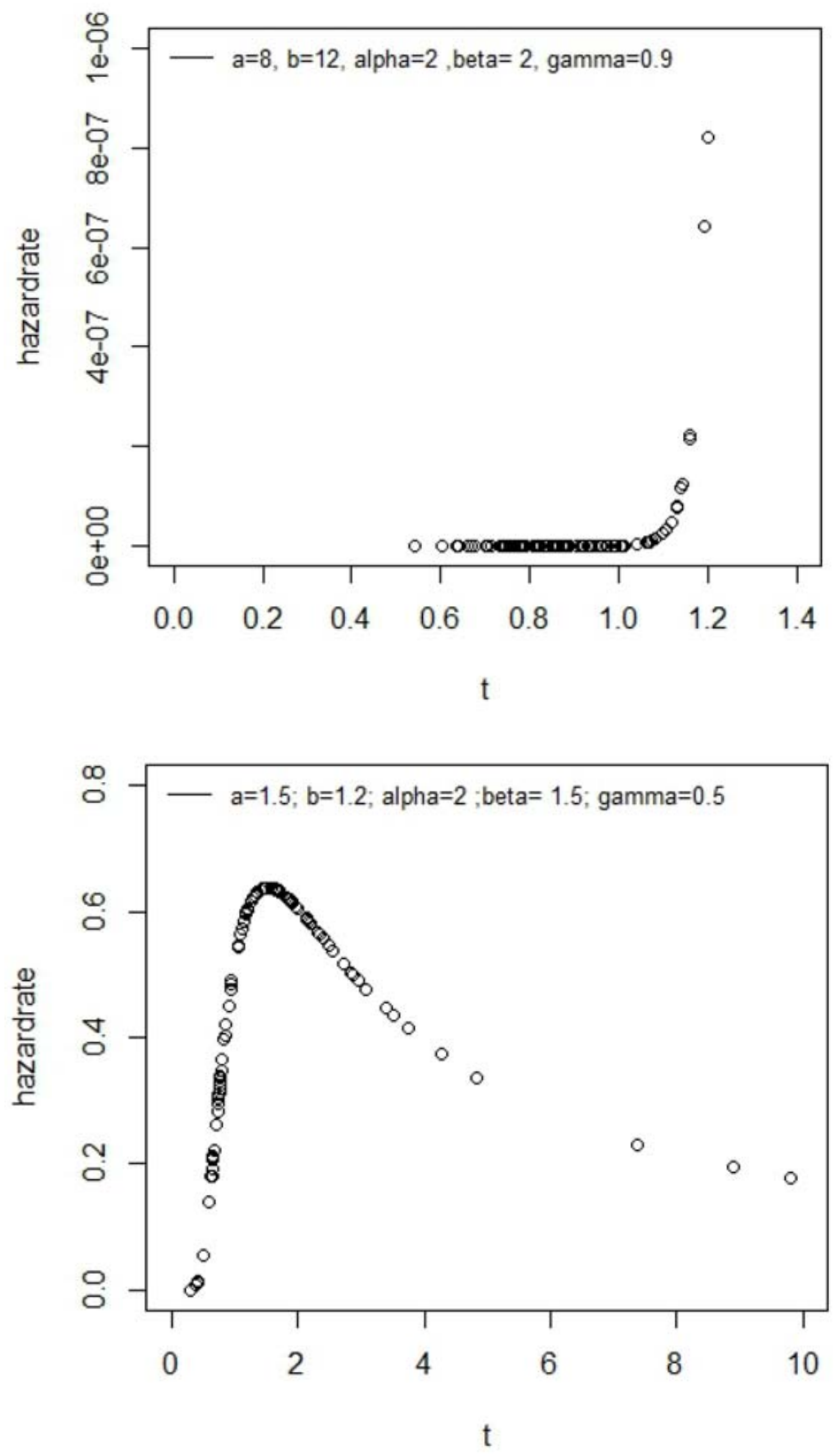


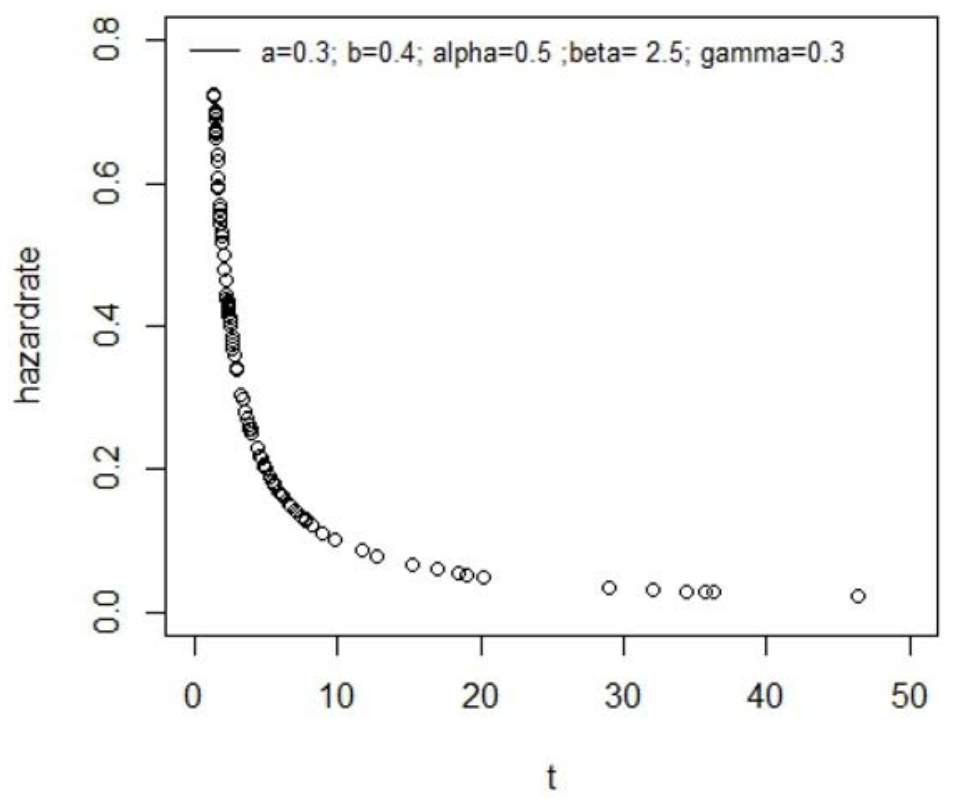

Figure 1. Different shapes of hazard rate function of the Kumaraswamy generalized inverse Weibull (Kum-GIW) distribution.

\section{Estimating the Parameters of the Kum-GIW Distribution with Censored Data}

Let $T$ be a random variable distributed with the vector of parameters $\theta=(a, b, \alpha, \beta, \gamma)^{T}$. Suppose that $T_{i}$ are failure times non-negative and independent and the probability density function of $T_{i}$ belongs to a parametric family (Kum-GIW in our case). The censoring times $C_{i}$ are also non-negative and assumed to be random sample.

Suppose that the data consist of $n$ independent observations $t_{i}=\min \left(T_{i}, C_{i}\right)$ for $i=1,2, \ldots, n$. The right censoring is non informative $\left(C_{i}\right.$ does not depend on $\left.\theta\right)$. So in this case, we obtain the following expressions of the likelihood functions: 


$$
\begin{aligned}
L(t, \theta) & =\prod_{i=1}^{n} f^{\delta_{i}}\left(t_{i}, \theta\right) S^{1-\delta_{i}}\left(t_{i}, \theta\right) \\
& =\prod_{i=1}^{n} \lambda^{\delta_{i}}\left(t_{i}, \theta\right) S\left(t_{i}, \theta\right), \quad \delta_{i}=1_{\left\{T_{i} \leq C_{i}\right\}} .
\end{aligned}
$$

The log-likelihood function for the Kum-GIW model is given by

$$
\begin{aligned}
l(t, \theta)= & \sum_{i=1}^{n} \delta_{i}\left[\ln a+\ln b+\ln \gamma+\ln \beta+\beta \ln \alpha-(\beta+1) \ln \left(t_{i}\right)-a \gamma\left(\frac{\alpha}{t_{i}}\right)^{\beta}\right. \\
& \left.-\ln \left(1-\exp \left\{-a \gamma\left(\frac{\alpha}{t_{i}}\right)^{\beta}\right\}\right)\right]+b \sum_{i=1}^{n} \ln \left(1-\exp \left\{-a \gamma\left(\frac{\alpha}{t_{i}}\right)^{\beta}\right\}\right), \\
l(t, \theta)= & m \ln a+m \ln b+m \ln \gamma+m \ln \beta+m \beta \ln \alpha-(\beta+1) \sum_{i \in F} \ln \left(t_{i}\right)-m a \gamma \alpha^{\beta} \sum_{i \in F} t_{i}^{-\beta} \\
+ & (b-1) \sum_{i \in F} \ln \left(1-\exp \left\{-a \gamma\left(\frac{\alpha}{t_{i}}\right)^{\beta}\right\}\right)+b \sum_{i \in C} \ln \left(1-\exp \left\{-a \gamma\left(\frac{\alpha}{t_{i}}\right)^{\beta}\right\}\right),
\end{aligned}
$$

where $F$ and $C$ are the sets of complete and censored observations, respectively, $m$ is the number of failures.

The maximum likelihood estimations (MLE's) $\hat{\theta}=(\widehat{a}, \hat{b}, \hat{\alpha}, \widehat{\beta}, \hat{\gamma})^{T}$ of the parameters are the solution of the non-linear system of score functions $\left(i_{\alpha}(\theta), i_{b}(\theta), i_{\alpha}(\theta), i_{\beta}(\theta), i_{\gamma}(\theta)\right)^{T}=\mathbf{0}_{5}$. (For more details, see the Appendix).

\section{Goodness-of-fit Test for Right Censored Data}

For testing the goodness-of-fit of a parametric family of survival distribution from right censored data, Habib and Thomas [11], Hollander and Pena [12] considered natural modifications of the Nikulin-RaoRobson (NRR) statistic for data without covariates. Also, Hjort [13], Hollander and Pena [12] considered goodness-of-fit for parametric Cox models, Gray and Pierce [10], Akritas [1], and Zhang [33] for linear regression models. 
Bagdonavicius and Nikulin [3] gave chi-squared type goodness-of-fittest for censored data with possibly time dependent covariates and considered random grouping intervals as data functions. These tests are based on maximum likelihood estimations for ungrouped data, and random grouping intervals are considered as data functions. We adapt this test for a Kumaraswamy generalized inverse Weibull model. Let us consider the hypothesis

$$
H_{0}: F(x) \in F_{0}=\left\{F_{0}(x, \theta), x \in R^{1}, \theta \in \Theta \subset R^{s}\right\}
$$

where $\theta=\left\{\theta_{1}, \ldots, \theta_{s}\right\}^{T} \in \Theta \subset R^{s}$ is unknown $s$-dimensional vector parameter and $F_{0}$ is a known distribution function. Let us consider a finite time interval only say $[0, \tau]$ and divide it into $k>s$ smaller intervals $I_{j}=\left(a_{j-1}, a_{j}\right]$, where

$$
0=<a_{0}<a_{1} \ldots<a_{k-1}<a_{k}=+\infty .
$$

In this case, the estimated $\widehat{a}_{j}$ is given by

$$
\widehat{a}_{j}=\Lambda^{-1}\left(\left(E_{j}-\sum_{l=1}^{i-1} \Lambda\left(X_{(l)}, \hat{\theta}\right)\right) /(n-i+1), \hat{\theta}\right), \quad \widehat{a}_{k}=X_{(n)}, j=1, \ldots, k
$$

where $\hat{\theta}$ is the maximum likelihood estimator of the parameter $\theta, \Lambda^{-1}$ is the inverse of the cumulative hazard function $\Lambda, X_{(i)}$ is the $i$-th element in the ordered statistics $\left(X_{(1)}, \ldots, X_{(n)}\right)$ and $E_{j}=(n-i+1) \Lambda\left(\widehat{a}_{j}, \hat{\theta}\right)+$ $\sum_{l=1}^{i-1} \Lambda\left(X_{(l)}, \hat{\theta}\right) ; a_{j}$ are random data functions such as the $k$ intervals chosen have equal expected numbers of failures $e_{j}$.

The test is based on the vector

$$
Z=\left(Z_{1}, \ldots, Z_{k}\right)^{T}, \quad Z_{j}=\frac{1}{\sqrt{n}}\left(U_{j}-e_{j}\right), \quad j=1,2, \ldots, k
$$

$U_{j}$ represent the numbers of observed failures in these intervals. 
Under the hypothesis $H_{0}$, for parametric models with survival functions and hazard rates absolutely continuous and non informative censoring, Bagdonavicius et al. [4] (Theorems 1 and 2) showed that the limit distribution of the statistic test

$$
Y^{2}=Z^{T} \hat{\Sigma}^{-} Z=\sum_{j=1}^{k} \frac{\left(U_{j}-e_{j}\right)^{2}}{U_{j}}+Q,
$$

where $\hat{\Sigma}^{-}$is a generalized inverse of the estimated covariance matrix of the vector $Z$,

$$
\widehat{\Sigma}^{-}=\widehat{A}^{-1}+\widehat{A}^{-1} \widehat{C}^{T} \widehat{G}^{-} \widehat{C} \widehat{A}^{-1}, \quad \widehat{G}=\widehat{I}-\widehat{C} \widehat{A}^{-1} \widehat{C}^{T},
$$

and

$$
\begin{aligned}
& Q=\widehat{W}^{T} \widehat{G}^{-} \widehat{W}, \quad \widehat{A}_{j}=U_{j} / n, \quad U_{j}=\sum_{i: X_{i} \in I_{j}} \delta_{i}, \\
& \widehat{W}=\left(\widehat{W}_{1}, \ldots, \widehat{W}_{s}\right)^{T}, \quad \widehat{G}=\left[\widehat{g}_{l l^{\prime}}\right]_{s \times s}, \quad \widehat{g}_{l l^{\prime}}=\widehat{i}_{l l^{\prime}}-\sum_{j=1}^{k} \widehat{C}_{l j} \widehat{C}_{l^{\prime} j} \hat{A}_{j}^{-1}, \\
& \widehat{C}_{l j}=\frac{1}{n} \sum_{i: X_{i} \in I_{j}} \delta_{i} \frac{\partial}{\partial \theta} \ln \lambda\left(t_{i}, \widehat{\theta}\right), \quad \widehat{i}_{l l^{\prime}}=\frac{1}{n} \sum_{i=1}^{n} \delta_{i} \frac{\partial \ln \lambda\left(t_{i}, \hat{\theta}\right)}{\partial \theta_{l}} \frac{\partial \ln \lambda\left(t_{i}, \hat{\theta}\right)}{\partial \theta_{l^{\prime}}}, \\
& \widehat{W}_{l}=\sum_{j=1}^{k} \widehat{C}_{l j} \widehat{A}_{j}^{-1} Z_{j}, \quad l, l^{\prime}=1, \ldots, s,
\end{aligned}
$$

is chi-square with $r=\operatorname{rank}\left(\Sigma^{-}\right)$degrees of freedom. The hypothesis is rejected with approximate significance level $\alpha$ if $Y_{n}^{2}>\chi_{\alpha}^{2}(r)$, where $\chi_{\alpha}^{2}(r)$ is the quantile of chi-square with $r$ degrees of freedom. For more details, see Bagdonavicius and Nikulin [2], Bagdonavicius and Nikulin [3]. 


\section{Validity of the Kum-GIW Model in Censoring Case}

The choice of $\widehat{a}_{j} ; j=1,2, \ldots, k$, in the case of a Kum-GIW model, is obtained as follows:

$$
\begin{gathered}
\widehat{a}_{j}=\left[-b \ln \left(1-\exp \left\{-a \gamma \alpha^{\beta}\left[E_{j}+b \sum_{l=1}^{i-1} \ln \left(1-\exp \left\{-a \gamma \alpha^{\beta} X_{(l)}^{-\beta}\right\}\right)\right]^{-\beta}\right\}(n-i+1)\right]\right]^{-1} \\
\widehat{a}_{k}=X_{(n)} .
\end{gathered}
$$

To obtain the explicit form of the modified chi-squared $Y_{n}^{2}$ statistic for the Kum-GIW model, we must calculate the matrix $\widehat{C}=\left[\widehat{c_{l j}}\right]_{5 \times 5}$ and the Fisher information matrix $\hat{\mathbf{i}}=\left[\widehat{i_{l l^{\prime}}}\right]_{5 \times 5}$. (For more details, see the Appendix).

\subsection{The matrix $\widehat{C}$}

The elements of the estimated matrix $\widehat{C}$ with $\widehat{C}=\frac{1}{n} \sum_{i: t \in I_{j}}^{n} \delta_{i}$ $\frac{\partial \ln \lambda\left(t_{i}, \theta\right)}{\partial \theta_{l}}, l=1,2, \ldots, m$ and $j=1,2, \ldots, k$

$$
\begin{gathered}
\widehat{C_{1 j}}=\sum_{i: t \in I_{j}}^{n} \delta_{i}\left[\frac{1-a \gamma\left(\frac{\alpha}{t_{i}}\right)^{\beta}}{a}-\frac{\gamma\left(\frac{\alpha}{t_{i}}\right)^{\beta} M\left(t_{i}, \theta\right)}{1-M\left(t_{i}, \theta\right)}\right], \\
\widehat{C_{2 j}}=\sum_{i: t \in I_{j}}^{n} \delta_{i} \frac{1}{b}, \\
\widehat{C_{3 j}}=\sum_{i: t \in I_{j}}^{n} \delta_{i}\left[\frac{1-a \gamma \alpha \beta \alpha^{\beta-1} t_{i}^{-\beta}}{\alpha}-\frac{a \gamma \beta \alpha^{\beta-1} t_{i}^{-\beta} M\left(t_{i}, \theta\right)}{1-M\left(t_{i}, \theta\right)}\right],
\end{gathered}
$$




$$
\begin{gathered}
\widehat{C_{4 j}}=\sum_{i: t \in I_{j}}^{n} \delta_{i}\left[\frac{1-\beta \ln \left(t_{i}\right)-\beta a \gamma\left(\frac{\alpha}{t_{i}}\right)^{\beta} \ln \left(\frac{\alpha}{t_{i}}\right)}{\beta}-\frac{a \gamma\left(\frac{\alpha}{t_{i}}\right)^{\beta} \ln \left(\frac{\alpha}{t_{i}}\right) M\left(t_{i}, \theta\right)}{1-M\left(t_{i}, \theta\right)}\right], \\
\widehat{C_{5 j}}=\sum_{i: t \in I_{j}}^{n} \delta_{i}\left[\frac{1-a \gamma\left(\frac{\alpha}{t_{i}}\right)^{\beta}}{\gamma}-\frac{a\left(\frac{\alpha}{t_{i}}\right)^{\beta} M\left(t_{i}, \theta\right)}{1-M\left(t_{i}, \theta\right)}\right] .
\end{gathered}
$$

So we can calculate the estimated matrix $\widehat{w}$ defined as

$$
\widehat{w_{l}}=\sum_{j=1}^{k}{\widehat{C_{l j}}}_{A_{j}}^{-1} Z_{j}, \quad l=1,2, \ldots, 5, \quad j=1,2, \ldots, k
$$

Then, we obtain the statistic $Y_{n}^{2}$ for the model of Kumaraswamy generalized inverse Weibull as follow:

$$
Y^{2}=\sum_{j=1}^{k} \frac{\left(U_{j}-e_{j}\right)^{2}}{U_{j}}+\widehat{W}^{T} \times\left[\hat{i}_{l l^{\prime}}-\sum_{j=1}^{k} \widehat{C}_{l j} \widehat{C}_{l^{\prime} j} \widehat{A}_{j}^{-1}\right]^{-} \times \widehat{W}, \quad l, l^{\prime}=1,2, \ldots, 5 .
$$

\section{Simulation Study}

\subsection{Kum-GIW's parameters estimation}

In order to evaluate the performance of the maximun likelihood method of estimating the Kum-GIW parameters in complete and censored case, we use the $\mathrm{R}$ statistical software and Brazilai-Bormein (BB) algorithms (Ravi et al. [28]). We give the mean simulated maximum likelihood estimators values $\hat{a}, \hat{b}, \widehat{\alpha}, \widehat{\beta}, \hat{\gamma}$ and their mean square errors in Table 1. Seven sample sizes $(n=100, n=150, n=200, n=250$, $n=300, n=350$, and $n=500$ ) are considered. The data were simulated $N=10,000$ with the following values of the parameters: $a=1.5, b=2.5$, $\alpha=2, \beta=1.5, \gamma=3$. 
Table 1. Mean simulated maximum likelihood estimators value and their mean square errors

\begin{tabular}{|c|c|c|c|c|c|c|c|}
\hline$N=10,000$ & $n=100$ & $n=150$ & $n=200$ & $n=250$ & $n=300$ & $n=350$ & $n=500$ \\
\hline$\widehat{a}$ & 1.6123 & 1.5745 & 1.5709 & 1.5217 & 1.5326 & 1.5109 & 1.5023 \\
\hline $\mathrm{SME}$ & 0.0042 & 0.0036 & 0.0034 & 0.0029 & 0.0031 & 0.0018 & 0.0006 \\
\hline$\widehat{b}$ & 2.7013 & 2.6398 & 2.6182 & 2.6187 & 2.6011 & 2.5434 & 2.5117 \\
\hline $\mathrm{SME}$ & 0.0053 & 0.0047 & 0.0044 & 0.0046 & 0.0028 & 0.0022 & 0.0007 \\
\hline$\widehat{\alpha}$ & 2.1662 & 2.1219 & 2.1067 & 2.1104 & 2.0971 & 2.0783 & 2.0122 \\
\hline $\mathrm{SME}$ & 0.0036 & 0.0028 & 0.0022 & 0.0017 & 0.0011 & 0.0009 & 0.0003 \\
\hline$\widehat{\beta}$ & 1.7005 & 1.6308 & 1.6017 & 1.5806 & 1.5472 & 1.5508 & 1.5051 \\
\hline $\mathrm{SME}$ & 0.0056 & 0.0048 & 0.0042 & 0.0031 & 0.0025 & 0.0028 & 0.0006 \\
\hline$\hat{\gamma}$ & 2.9574 & 2.9525 & 2.9765 & 2.9963 & 2.9904 & 3.0819 & 3.0057 \\
\hline $\mathrm{SME}$ & 0.0041 & 0.0038 & 0.0026 & 0.0018 & 0.0021 & 0.0013 & 0.0004 \\
\hline
\end{tabular}

From Table 1, we can note that the maximum likelihood method of estimating the Kum-GIW parameters is well perform.

The simulated average absolute errors of MLE $\hat{\theta}=(\hat{a}, \hat{b}, \hat{\alpha}, \widehat{\beta}, \hat{\gamma})^{T}$ versus their true value can be find in Table 2 . For each simulated sample $n$, we compute the average absolute of errors as the total of absolute different of the MLE's against the true value of the number of runs $N=10,000$ times. 
Table 2. Simulate average absolute errors of MLE $\hat{\theta}$ versus their true value $\theta=(a=1.5, b=2.5, \alpha=2, \beta=1.5, \gamma=3)^{T}$ when data is completed $(p=0)$ and the right censoring probability $p=20 \%$

\begin{tabular}{|c|c|c|c|c|c|c|c|c|c|c|c|}
\hline$n$ & $\simeq n^{0.5}$ & $\widehat{a}$ & $\widehat{a}$ & $\hat{b}$ & $\hat{b}$ & $\hat{\alpha}$ & $\hat{\alpha}$ & $\hat{\beta}$ & $\widehat{\beta}$ & $\hat{\gamma}$ & $\hat{\gamma}$ \\
\hline & & $p=0 \%$ & $p=20 \%$ & $p=0 \%$ & $p=20 \%$ & $p=0 \%$ & $p=20 \%$ & $p=0 \%$ & $p=20 \%$ & $p=0 \%$ & $p=20 \%$ \\
\hline 100 & 0.1210 & 0.0542 & 0.0627 & 0.0471 & 0.0515 & 0.0584 & 0.0603 & 0.0551 & 0.0610 & 0.0522 & 0.0592 \\
\hline 150 & 0.0946 & 0.0412 & 0.0587 & 0.0462 & 0.0494 & 0.0478 & 0.0593 & 0.0444 & 0.0562 & 0.0411 & 0.0475 \\
\hline 200 & 0.0865 & 0.0405 & 0.0418 & 0.0385 & 0.0478 & 0.0402 & 0.0482 & 0.0391 & 0.0439 & 0.0365 & 0.0391 \\
\hline 250 & 0.0753 & 0.0361 & 0.0382 & 0.0326 & 0.0466 & 0.0376 & 0.0377 & 0.0302 & 0.0386 & 0.0284 & 0.0274 \\
\hline 300 & 0.6078 & 0.0300 & 0.0354 & 0.0298 & 0.0385 & 0.0312 & 0.0304 & 0.0290 & 0.0304 & 0.0205 & 0.0209 \\
\hline 350 & 0.4526 & 0.0265 & 0.0330 & 0.0284 & 0.0307 & 0.0209 & 0.0276 & 0.0281 & 0.0295 & 0.0193 & 0.0168 \\
\hline 500 & 0.4281 & 0.0244 & 0.0328 & 0.0279 & 0.0284 & 0.0154 & 0.0248 & 0.0273 & 0.0284 & 0.0169 & 0.0168 \\
\hline
\end{tabular}


Table 2 show that all simulated MLE converge faster than $n^{0.5}$ and, hence confirm the theorem established by Fisher [8], so we demonstrate that the MLE of Kum-GIW distribution is $\sqrt{n}$-consistent in the cases of complete and censored data.

\subsection{Simulated distribution of $Y_{n}^{2}$ statistic for Kum-GIW model}

We test the hypothesis $H_{0}$ that the statistic $Y_{n}^{2}$ follows a chisquared distribution with $k=12$ degrees of freedom. For that, we run 10,000 simulations of samples data (sample sizes $n=50, n=100, n=300$, and $n=500)$ from a Kum-GIW distribution. We group in Table 3 the number of rejection's cases of the hypothesis $H_{0}$, when $Y_{n}^{2}>\chi_{\alpha}^{2}(k)$ with $\alpha(\alpha=1 \%, \alpha=5 \%, \alpha=10 \%)$ significance levels.

Table 3. Simulated levels of significance for $Y_{n}^{2}(\hat{\theta})$ test against their theoretical values $(\alpha=0,01,0,05,0,1)$

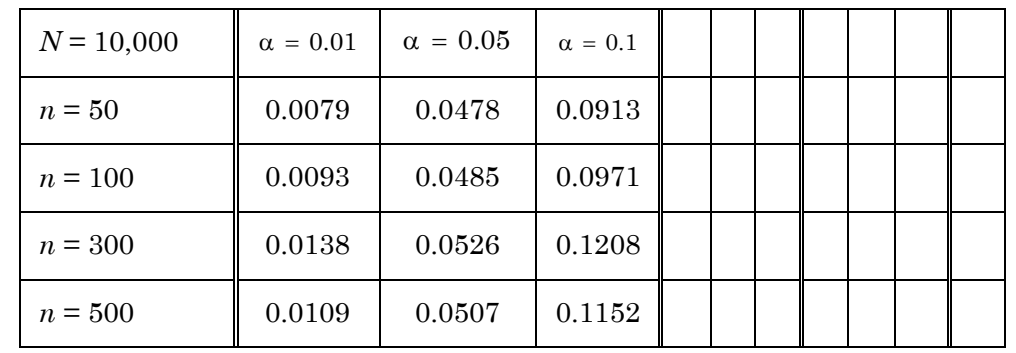

One can observe that theoretical levels of the chi-squared distributions with $k=12$ degrees of freedom matches with the simulated levels of significance for $Y_{n}^{2}$ test. Hence, we can say that the test proposed can adjust data from a Kumaraswamy generalized inverse Weibull distributions in acceptable manner.

For demonstrating that the $Y_{n}^{2}$ statistic follows in the limit; a chisquared distribution with $k$ degrees of freedom; we compute $N=10,000$ times, the simulated distribution of $Y_{n}^{2}(\hat{\theta})$ under the null hypothesis $H_{0}$ 
with different values of parameters $\operatorname{Kum}-\operatorname{GIW}(a, b, \alpha, \beta, \gamma)$; for example, $\widehat{\theta}=(\widehat{a}=1.5, \hat{b}=2.5, \widehat{\alpha}=2, \widehat{\beta}=1.5, \hat{\gamma}=3), \widehat{\theta}=(\widehat{a}=2.5, \hat{b}=2.5, \hat{\alpha}=2$, $\widehat{\beta}=1.5, \hat{\gamma}=3), \hat{\theta}=(\hat{a}=1.5, \hat{b}=2.5, \hat{\alpha}=1.5, \widehat{\beta}=1.5, \hat{\gamma}=3), \hat{\theta}=(\widehat{a}=1.5$, $\widehat{b}=1.5, \widehat{\alpha}=2, \hat{\beta}=1.5, \hat{\gamma}=3), \hat{\theta}=(\widehat{a}=1.5, \hat{b}=2.5, \widehat{\alpha}=2, \widehat{\beta}=0.5, \hat{\gamma}=3)$, $\widehat{\theta}=(\widehat{a}=1.5, \hat{b}=2.5, \widehat{\alpha}=2, \widehat{\beta}=1.5, \hat{\gamma}=2.5)$ and $r=13$ intervals, versus the chi-squared distribution with $k=r-1=12$ degree of freedom. Their histograms are represented in Figure 2 versus the chi-squared distribution with $k$ degree of freedom.

As can see from Figure 2, one can observe that the statistical distribution of $Y_{n}^{2}$ with different values of parameters and different numbers $k$ of grouping cells; in the limit follows a chi-squared with $r=k$ degrees of freedom within the statistical errors of simulation. We obtain the same results for different value of parameters and different number of equiprobable grouping intervals. It is means that the limiting distribution of the generalized chi-squared $Y_{n}^{2}$ statistic is distribution free. This result also established the theorem of Nikulin [17, 18, 19]. 


$$
\hat{a}=1.5, \hat{b}=2.5, \hat{\alpha}=2, \hat{\beta}=1.5, \hat{\gamma}=3
$$

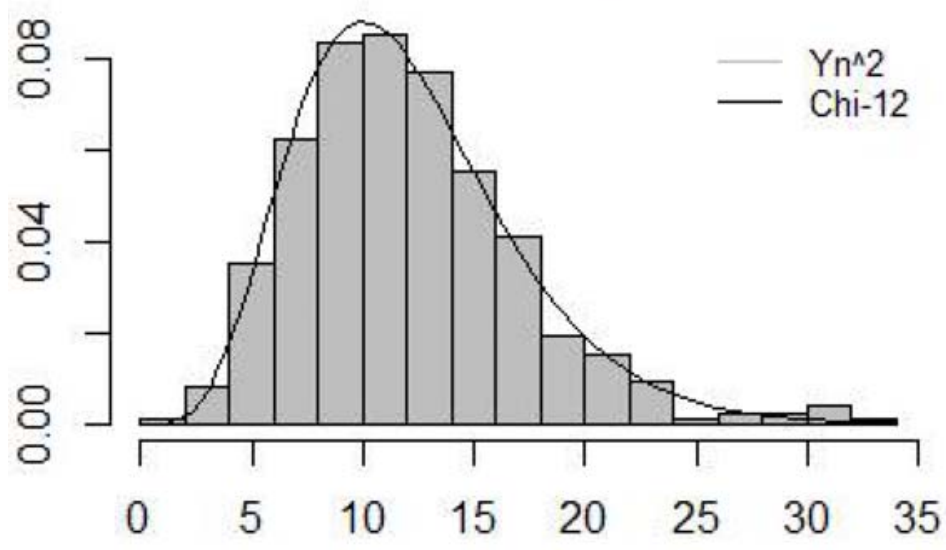

$$
\hat{a}=2.5, \hat{b}=2.5, \hat{\alpha}=2, \hat{\beta}=1.5, \hat{\gamma}=3
$$

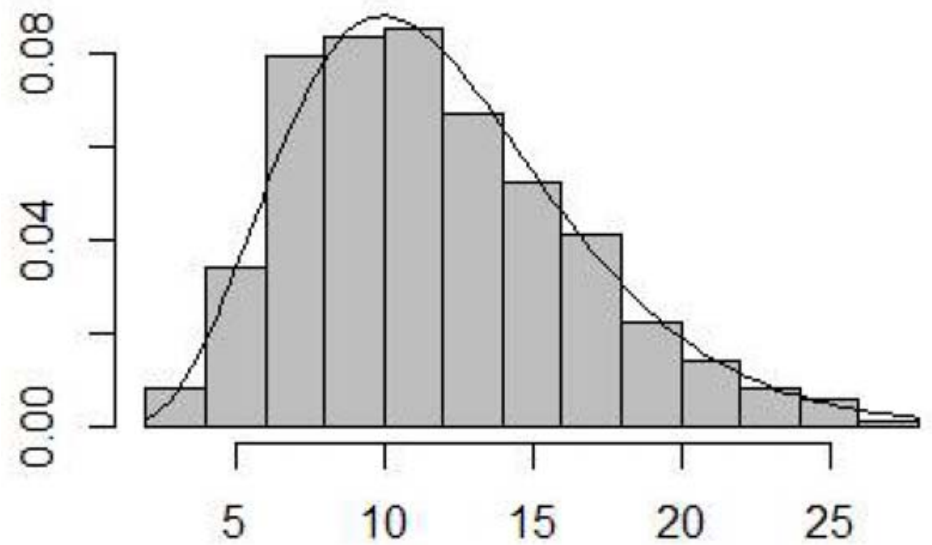




$$
\hat{a}=1.5, \hat{b}=2.5, \hat{\alpha}=1.5, \hat{\beta}=1.5, \hat{\gamma}=3
$$

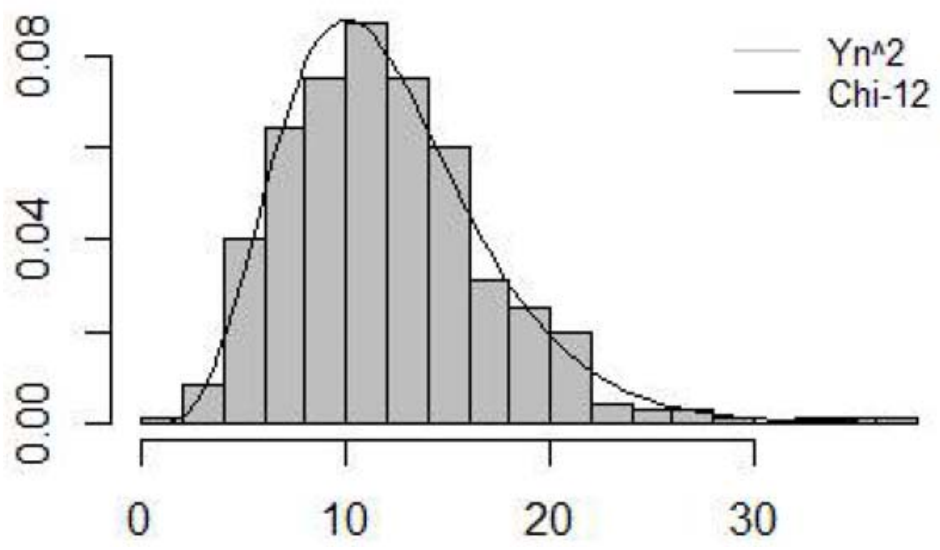

$$
\hat{a}=1.5, \hat{b}=1.5, \hat{\alpha}=2, \hat{\beta}=1.5, \hat{\gamma}=3
$$

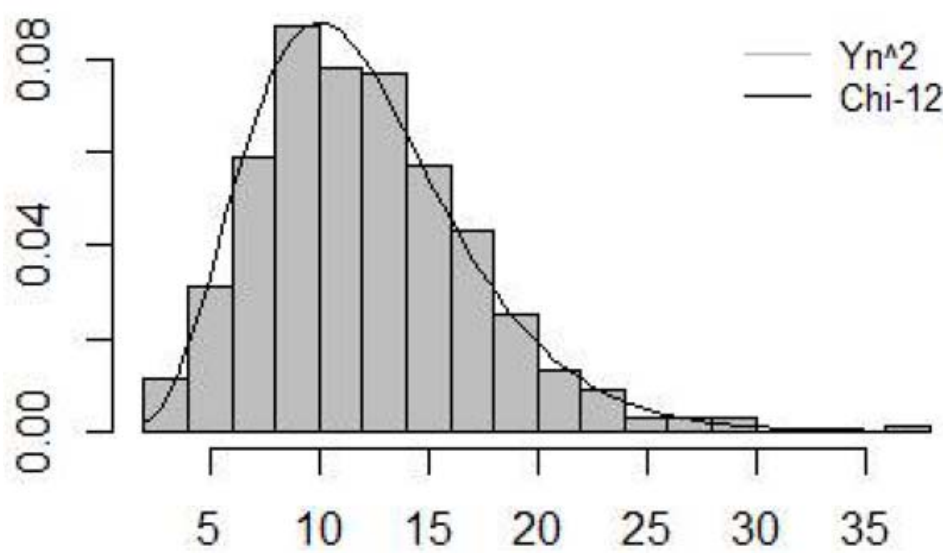




$$
\hat{a}=1.5, \hat{b}=2.5, \hat{\alpha}=2, \hat{\beta}=0.5, \hat{\gamma}=3
$$

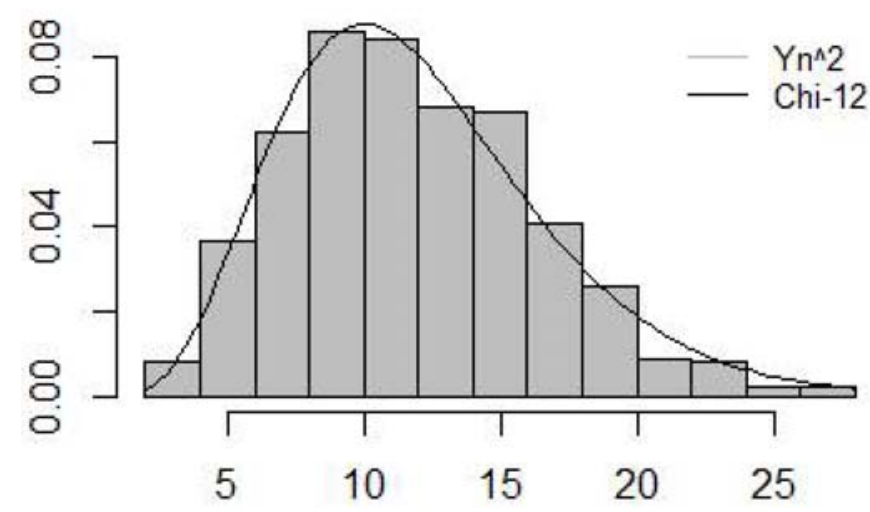

$$
\hat{a}=1.5, \hat{b}=2.5, \hat{\alpha}=2, \hat{\beta}=1.5, \hat{\gamma}=2.5
$$

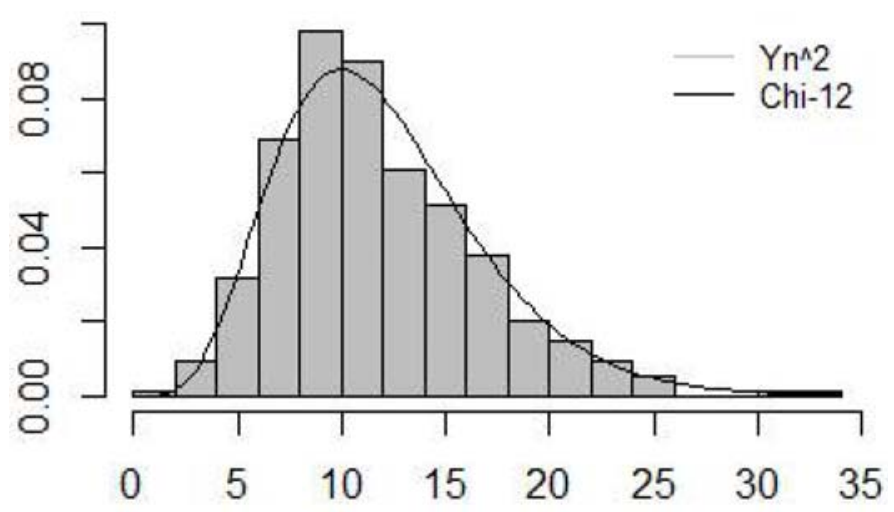

Figure 2. Simulated distribution of the $Y_{n}^{2}$ statistic under the null hypothesis $H_{0}$, with different parameters of $\hat{\theta}$ versus the chi-squared distribution with 12 degrees of freedom, with $n=150, N=10,000$.

\subsection{Application to real data}

To show the application of the Kumaraswamy generalized inverse Weibull model to survival and reliability data in the case of censored data, we use two real life data sets by using $Y_{n}^{2}$ statistic. 


\subsection{Survival analysis data case}

Pike [27] gave some data from a laboratory investigation in which the vaginas of rats were painted with the carcinogen DMBA, and the number of days $T$ until a carcinoma appeared was recorded. The data below are for a group of 19 rats (Group 1 in Pike's paper); the two observations with asterisks are censoring times.

$143,164,188,188,190,192,206,209,213,216,220,227,230,234,246$, $265,304,216^{*}, 244^{*}$.

These data are analyzed by Nikulin and Tran [25]; where they suggested that the probability plots for two parameters Weibull distributions, then the data are best fitting with generalized BirnbaumSaunders, logistic and the generalized Birnbaum-Saunders cauchy distributions.

We consider the hypothese that these data follow a Kum-GIW distribution. Choosing $k=6$, grouping intervals, we find the values of the maximum likelihood estimators parameters:

$$
\widehat{a}=0.5712, \widehat{b}=2.3884, \widehat{\alpha}=4.0187, \widehat{\beta}=0.1287, \hat{\gamma}=3.8854
$$

The values of $\widehat{a}_{j}$, the frequency vector $Z$ are given in the following table:

\begin{tabular}{|c|c|c|c|c|c|c|}
\hline$j$ & 1 & 2 & 3 & 4 & 5 & 6 \\
\hline$\widehat{a}_{j}$ & 188.51 & 194.38 & 214.76 & 219.85 & 240.77 & 304 \\
\hline$U_{J}$ & 4 & 2 & 3 & 2 & 4 & 4 \\
\hline$e_{j}$ & 35.268 & 35.268 & 35.268 & 35.268 & 35.268 & 35.268 \\
\hline$Z_{j}$ & -7.1734 & -7.6322 & -7.6325 & -7.632202 & -7.1734 & -7.1734 \\
\hline
\end{tabular}

The result of the elements of the $Y_{n}^{2}$ statistic are

$$
\widehat{W}=\left[\begin{array}{llll}
0.9815 & 1214.0208 & -64.8294 & -0.0038
\end{array}\right],
$$

and the estimated Fisher's information matrix $\widehat{I}_{5 \times 5}$ is 


$$
\widehat{I}=\left(\begin{array}{c}
3.2806 \times 10^{-5} \\
2.5512 \times 10^{-2} \\
-5.0102 \times 10^{-3} \\
1.8726 \times 10^{-4} \\
-2.0043 \times 10^{-3}
\end{array}\right.
$$

and

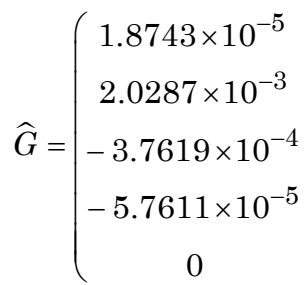

$$
\begin{array}{cc}
2.5512 \times 10^{-2} & -5.0102 \times 10^{-3} \\
1.2296 \times 10^{-3} & -12.4398 \\
-12.4398 & 5.3286 \times 10^{-2} \\
3.3356 \times 10^{-4} & -243.85 \\
5.1083 \times 10^{-4} & -1.2096 \times 10^{-2}
\end{array}
$$

$$
\begin{aligned}
& 2.0287 \times 10^{-3} \\
& 4.4381 \times 10^{-5} \\
& -34.8754 \times 10^{-2} \\
& 1.8753 \times 10^{-4} \\
& 2.0598 \times 10^{-5}
\end{aligned}
$$

$$
\begin{gathered}
-3.7619 \times 10^{-4} \\
-34.8754 \times 10^{-2} \\
-204.37 \\
-561.712 \times 10^{-1} \\
-14.784 \times 10^{-2}
\end{gathered}
$$

$$
\begin{gathered}
1.8726 \times 10^{-4} \\
3.3356 \times 10^{-4} \\
-243.85 \\
-1.2388 \times 10^{-1} \\
0
\end{gathered}
$$

$$
\left.\begin{array}{c}
-2.0043 \times 10^{-3} \\
5.1083 \times 10^{-4} \\
-1.2096 \times 10^{-2} \\
0 \\
0
\end{array}\right) \text {, }
$$

$$
\begin{gathered}
-5.7611 \times 10^{-5} \\
1.8753 \times 10^{-4} \\
-561.712 \times 10^{-1} \\
-8.281 \times 10^{-3} \\
0
\end{gathered}
$$


By using the NRR statistic for five parameters Kumaraswamy generalized inverse Weibull distribution, we obtain the value $Y_{n}^{2}=11.9589$. Since $Y_{n}^{2}=11.9589<\chi_{0.05}^{2}(6)=12.5916$ (the critical value), we can conclude that the Kumaraswamy generalized inverse Weibull (Kum-GIW) model is in concordance with the appeared time of the carcinoma of Pike.

\subsection{Reliability data (cycles-to-failure of cylindrical steel specimens)}

Cycles-to-failure of cylindrical steel specimens of $41 \% \mathrm{C}$ anneal for 30 min at $870^{\circ} \mathrm{C}$; after surface finish with coarse emery, tested by Ono's Rotary Uniform Bending Tester at 2000rpm with $\pm 35.6 \mathrm{~kg} / \mathrm{mm}^{2}$. This data is obtained from Lawless [16]

$\begin{array}{ccccccccc}44.9 & 50.5 & 53.0 & 58.3 & 67.7 & 71.5 & 82.1 & 91.3 & 104.6 \\ 119.9 & 122.8 & 123.7 & 138.9 & 141.0 & 142.8 & 143.1 & 145.3 & 147.8 \\ 151.8 & 157.2 & 163.2 & 172.8 & 180.5 & 185.3 & 188.1 & 192.1 & 202.0 \\ 213.2 & 223.6 & 240.2 & 243.8 & 244.4 & 284.4 & 287.2 & 298.8 & 324.7 \\ 330.3 & 350.1 & 354.5 & 380.5 & 385.8 & 392.0 & 394.1 & 406.6 & 423.0 \\ 423.3 & 443.6 & 481.2 & 548.2 & 671.1 & 740.7 & 748.7 & 815.6 & 819.5 \\ 834.7 & 967.9 & 1190.8 & 1286.6 & 1315.7 & 1379.3 & 1691.7 & 2187.8 & 2272.2 \\ 2505.9 & 2580.7 & 3643.5 & 3694.0 & 4212.7 . & & & & \end{array}$

The MLE's of the parameters $\widehat{a}, \hat{b}, \hat{\alpha}, \widehat{\beta}, \hat{\gamma}$ of the Kumaraswamy generalized inverse Weibull (Kum-GIW) model are

$$
\widehat{a}=0.7643, \widehat{b}=0.1287, \widehat{\alpha}=4.5342, \widehat{\beta}=1.7643, \widehat{\gamma}=2.0983 .
$$

If we choose $k=8$ intervals, the following table gives the results

\begin{tabular}{|c|c|c|c|c|c|c|c|c|}
\hline$j$ & 1 & 2 & 3 & 4 & 5 & 6 & 7 & 8 \\
\hline$\widehat{a}_{j}$ & 69.06 & 130.94 & 146.72 & 183.64 & 286.13 & 423.1 & 1264.93 & 4212.7 \\
\hline$U_{J}$ & 5 & 7 & 5 & 6 & 10 & 12 & 12 & 11 \\
\hline$e_{j}$ & 56.48 & 56.48 & 56.48 & 56.48 & 56.48 & 56.48 & 56.48 & 56.48 \\
\hline$Z_{j}$ & -6.242866 & -6.000331 & -6.242866 & -6.121599 & -5.636527 & -5.393992 & -5.393992 & -5.515260 \\
\hline
\end{tabular}


The statistical inferences for testing the null hypotheses that acute cycles-to-failure of cylindrical steel specimens belong the Kum-GIW model, are given as follows:

$$
\widehat{W}=\left[\begin{array}{llll}
1.5781 & -6.8714 & 123.6598 & -12.9377
\end{array}\right],
$$

and the estimated Fisher's information matrix is 


$\widehat{I}=\left(\begin{array}{ccccc}3.2806 \times 10^{-3} & -3.1893 \times 10^{-4} & -123.7601 \times 10^{-5} & -14.3387 \times 10^{-2} & 1.9802 \times 10^{-3} \\ -3.1893 \times 10^{-4} & 4.0942 \times 10^{-5} & -6.8631 \times 10^{-2} & -1298 \times 10^{-4} & -5.1083 \times 10^{-4} \\ -123.7601 \times 10^{-5} & -6.8631 \times 10^{-2} & -.2153 \times 10^{-3} & -402.89 & -3.0029 \times 10^{-5} \\ -14.3387 \times 10^{-2} & -1298 \times 10^{-4} & -402.89 & -1.9267 \times 10^{-1} & 201.3871 \\ 1.9802 \times 10^{-3} & -5.1083 \times 10^{-4} & -3.0029 \times 10^{-5} & 201.3871 & 0\end{array}\right)$.


We obtain $X^{2}=10.6574$ and $Q=4.55348$, so the value of the statistic test is $Y_{n}^{2}=15.2109$. Since $Y_{n}^{2}=15.2109<\chi_{0.05}^{2}(8)=15.50731$ (the critical value), the hypothesis $H_{0}$ of the Kumaraswamy generalized inverse Weibull (Kum-GIW) distribution is well accepted at the significance level $\alpha=0.05$.

\section{References}

[1] M. G. Akritas, Pearson-type goodness-of-fit tests: The univariate case, Journal of the American Statistical Association 83 (1988), 222-230.

[2] V. Bagdonavicius and M. Nikulin, Chi-squared tests for general composite hypotheses from censored samples, Comptes Rendus Mathematique 349(3) (2011), 219-223.

[3] V. Bagdonavicius and M. Nikulin, Chi-squared goodness-of-fit test for right censored data, International Journal of Applied Mathematics and Statistics 4(SI-11A) (2011), 30-50.

[4] V. Bagdonavicius et al., Non-parametric Tests for Censored Data, Wiley, New York, 2013.

[5] G. M. Cordeiro, E. M. M. Ortega and S. Nadarajah, The Kumaraswamy Weibull distribution with application to failure data, J. Franklin Inst. 349 (2010), 1174-1197.

[6] G. M. Cordeiro and M. de Castro, A new family of generalized distributions, Journal of Statistical Computation and Simulation 81 (2011), 883-898.

[7] Felipe R. S. de Gusmão, M. Edwin, M. Ortega Gauss and M. Cordeiro, The generalized inverse Weibull distribution, Stat. Papers 52(3) (2009), 591-619.

[8] R. A. Fisher and others, On a property connecting the $\chi^{2}$ measure of discrepancy with the method of maximum likelihood, Journal of the Royal Statistical Society 87 (1928), 442-449.

[9] H. Goual and N. Seddik-Ameur, Chi-squared type test for the AFT-generalized inverse Weibull distribution, Communications in Statistics-Theory and Methods 43(13) (2014), 2605-2617.

[10] Robert J. Gray and Donald A. Pierce, Goodness-of-fit tests for censored survival data, Ann. Statist. 13(2) (1985), 552-563.

[11] M. G. Habib and D. R. Thomas, Chi-square goodness-if-fit tests for randomly censored data, The Annals of Statistics 14(2) (1986), 759-765.

[12] M. Hollander and E. Pena, Chi-square goodness-of-fit test for randomly censored data, JASA 87(418) (1992), 458-463. 
[13] N. L. Hjort, Goodness of fit tests in models for life history data based on cumulative hazard rates, Ann. Statist. 18 (1990), 1221-1258.

[14] P. Kumaraswamy, Generalized probability density-function for double-bounded random-processes, Journal of Hydrology 462 (1980), 79-88.

[15] J. F. Lawless, Statistical Models and Methods for Lifetime Data (Second Edition), Wiley, New York, 2003.

[16] J. F. Lawless, Reliability, Life Testing and the Prediction of Service Lives: For Engineers and Scientists by Sam C. Saunders, Springer Series in Statistics 75(2) (2007), 260.

[17] L. Gerville-Réache, M. S. Nikulin, R. Tahir et al., On reliability approach and statistical inference in demography, Journal of Reliability and Statistical Studies 5 (2012), 37-49.

[18] S. Nadarajah, G. M. Cordeiro and E. M. M. Ortega, General results for the Kumaraswamy-G distribution, Journal of Statistical Computation and Simulation 82(7) (2012), 951-979.

[19] M. S. Nikulin, L. Gerville-Réache and X. Q. Tran, On chi-squared goodness-of-fit test for normality, In V. Couallier, L. Gerville-Réache, H. Huber-Carol, N. Limnios, and M. Mesbah, Editors, Statistical Models and Methods for Reliability and Survival Analysis, Wiley Online Library, New York, (2013), 213-227.

[20] M. S. Nikulin, Chi-squared test for normality, in proceedings of the International Vilnius Conference on Probability Theory and Mathematical Statistics 2 (1973a), 119-122.

[21] M. S. Nikulin, Chi-squared test for continuous distributions with shift and scal parameters, Theory of Probability and its Applications 18 (1973b), 559-568.

[22] M. S. Nikulin, On a chi-squared test for continuous distributions, Theory of Probability and its Applications 19 (1973c), 638-639.

[23] M. Nikulin and F. Haghighi, A chi-squared test for the generalized power weibull family for the head-and-neck cancer censored data, Journal of Mathematical Sciences 133(3) (2006), 1333-1341.

[24] M. S. Nikulin and X. Q. Tran, On chi-squared testing in accelerated trials, International Journal of Performability Engineering 10(1) (2014), 53-62.

[25] M. S. Nikulin and X. Q. Tran, Chi-squared goodness-of-fit tests for generalized Birnbaum-Saunders models for right censored data and its reliability applications, Journal of Reliability: Theory \& Application, Electronic Journal of International Groups on Reliability 8(\#2) (2013), 7-20.

[26] M. A. R. Pascoa, E. M. M. Ortega and G. M. Cordeiro, The Kumaraswamy generalized gamma distribution with application in survival analysis, Statistical Methodology 8 (2011), 411-433.

[27] M. C. Pike, A method of analysis of a certain class of experiments in carcinogenesis, Biometrics 22 (1966), 142-161. 
[28] V. Ravi and D. Gilbert Paul, BB: An R package for solving a large system of nonlinear equations and for optimizing a high-dimensional nonlinear objective function, Journal of Statistical Software 32(i04) (2009), 1-26.

[29] H. Saulo, J. Leao and M. Bourguignion, The Kumaraswamy birnbaum-sanders distribution, J. Stat. Theory Pract. 6 (2012), 754-759.

[30] M. Q. Shahbaz, S. Shahbaz and N. S. Butt, The Kumaraswamy inverse-Weibull distribution, Pakistan J. Statist. Oper. Res. 8 (2012), 479-489.

[31] V. Voinov, M. S. Nikulin and N. Balakrishnan, Chi-Squared Goodness of Fit Tests with Applications, Academic Press, New York, 2013.

[32] V. Voinov, N. Pya, N. Shapakov and Y. Voinov, Goodness-of-fit tests for the power generalized Weibull probability distribution, Communications in StatisticsSimulation and Computation 42(5) (2013), 1003-1012.

[33] B. Zhang, A chi-squared goodness-of-fit test for logistic regression models based on casecontrol data, Biometrika 86(3) (1999), 531-539. 


\section{Appendix}

The score functions are

$$
\begin{aligned}
& \dot{l}_{a}(\theta)=\frac{\partial l(t, \theta)}{\partial a}=\frac{r}{a}-r \gamma \alpha^{\beta} \sum_{i \in F} t_{i}^{-\beta}+(b-1) \sum_{i \in F} \frac{\gamma\left(\frac{\alpha}{t_{i}}\right)^{\beta} M\left(t_{i}, \theta\right)}{1-M\left(t_{i}, \theta\right)} \\
& +b \sum_{i \in C} \frac{\gamma\left(\frac{\alpha}{t_{i}}\right)^{\beta} M\left(t_{i}, \theta\right)}{1-M\left(t_{i}, \theta\right)} \\
& \dot{l}_{b}(\theta)=\frac{\partial l(t, \theta)}{\partial b}=\frac{r}{b}+\sum_{i \in F} \ln \left(1-M\left(t_{i}, \theta\right)\right)+\sum_{i \in C} \ln \left(1-M\left(t_{i}, \theta\right)\right), \\
& i_{\alpha}(\theta)=\frac{\partial l(t, \theta)}{\partial \alpha}=\frac{r}{\alpha}-\operatorname{ra\gamma } \beta \alpha^{\beta-1} \sum_{i \in F} t_{i}^{-\beta}+(b-1) \sum_{i \in F} \frac{a \gamma \beta \alpha^{\beta-1} t_{i}^{-\beta} M\left(t_{i}, \theta\right)}{1-M\left(t_{i}, \theta\right)} \\
& +b \sum_{i \in C} \frac{a \gamma \beta \alpha^{\beta-1} t_{i}^{-\beta} M\left(t_{i}, \theta\right)}{1-M\left(t_{i}, \theta\right)} \\
& \dot{l}_{\beta}(\theta)=\frac{\partial l(t, \theta)}{\partial \beta}=\frac{r}{\beta}-\sum_{i \in F} \ln \left(t_{i}\right)-\operatorname{ra\gamma } \alpha^{\beta} \sum_{i \in F} t_{i}^{-\beta}\left(\ln (\alpha)-\ln \left(t_{i}\right)\right) \\
& +(b-1) \sum_{i \in F} \frac{a \gamma \alpha^{\beta} t_{i}^{-\beta}\left[\ln (\alpha)-\ln \left(t_{i}\right)\right] M\left(t_{i}, \theta\right)}{1-M\left(t_{i}, \theta\right)} \\
& +b \sum_{i \in C} \frac{a \gamma \alpha^{\beta} t_{i}^{-\beta}\left[\ln (\alpha)-\ln \left(t_{i}\right)\right] M\left(t_{i}, \theta\right)}{1-M\left(t_{i}, \theta\right)}, \\
& i_{\gamma}(\theta)=\frac{\partial l(t, \theta)}{\partial \gamma}=\frac{r}{\gamma}-r a \alpha^{\beta} \sum_{i \in F} t_{i}^{-\beta}+(b-1) \sum_{i \in F} \frac{a\left(\frac{\alpha}{t_{i}}\right)^{\beta} M\left(t_{i}, \theta\right)}{1-M\left(t_{i}, \theta\right)}+b \sum_{i \in C} \frac{a\left(\frac{\alpha}{t_{i}}\right)^{\beta} M\left(t_{i}, \theta\right)}{1-M\left(t_{i}, \theta\right)}, \\
& \text { where } M\left(t_{i}, \theta\right)=\exp \left\{-a \gamma\left(\frac{\alpha}{t_{i}}\right)^{\beta}\right\} \text {. }
\end{aligned}
$$




\section{Fisher information matrix $\hat{\mathbf{i}}$}

The elements of the Fisher information matrix

$$
\begin{aligned}
& \hat{\mathbf{i}}=\left[\widehat{i_{l l^{\prime}}}\right] \text {, with } \widehat{i_{l l^{\prime}}}=\frac{1}{n} \sum_{i=1}^{n} \delta_{i} \frac{\partial \ln \lambda(t, \theta)}{\partial \theta_{l}} \frac{\partial \ln \lambda(t, \theta)}{\partial \theta_{l^{\prime}}} \text { are } \\
& \widehat{i_{11}}=\frac{1}{n} \sum_{i=1}^{n} \delta_{i}\left[\frac{1-a \gamma\left(\frac{\alpha}{t_{i}}\right)^{\beta}}{a}-\frac{\gamma\left(\frac{\alpha}{t_{i}}\right)^{\beta} M\left(t_{i}, \theta\right)}{1-M\left(t_{i}, \theta\right)}\right]^{2} \\
& \widehat{i_{12}}=\frac{1}{n} \sum_{i=1}^{n} \delta_{i}\left[\frac{1}{b} \frac{1-a \gamma\left(\frac{\alpha}{t_{i}}\right)^{\beta}}{a}-\frac{\gamma\left(\frac{\alpha}{t_{i}}\right)^{\beta} M\left(t_{i}, \theta\right)}{1-M\left(t_{i}, \theta\right)}\right] \text {, } \\
& \widehat{i_{13}}=\frac{1}{n} \sum_{i=1}^{n} \delta_{i}\left[\frac{1-a \gamma\left(\frac{\alpha}{t_{i}}\right)^{\beta}}{a}-\frac{\gamma\left(\frac{\alpha}{t_{i}}\right)^{\beta} M\left(t_{i}, \theta\right)}{1-M\left(t_{i}, \theta\right)}\right]\left[\frac{1-a \gamma \alpha \beta \alpha^{\beta-1} t_{i}^{-\beta}}{\alpha}-\frac{a \gamma \beta \alpha^{\beta-1} t_{i}^{-\beta} M\left(t_{i}, \theta\right)}{1-M\left(t_{i}, \theta\right)}\right] \text {, } \\
& \widehat{i_{14}}=\frac{1}{n} \sum_{i=1}^{n} \delta_{i}\left[\frac{1-a \gamma\left(\frac{\alpha}{t_{i}}\right)^{\beta}}{a}-\frac{\gamma\left(\frac{\alpha}{t_{i}}\right)^{\beta} M\left(t_{i}, \theta\right)}{1-M\left(t_{i}, \theta\right)}\right] \\
& \times\left[\frac{1-\beta \ln \left(t_{i}\right)-\beta a \gamma\left(\frac{\alpha}{t_{i}}\right)^{\beta} \ln \left(\frac{\alpha}{t_{i}}\right)}{\beta}-\frac{a \gamma\left(\frac{\alpha}{t_{i}}\right)^{\beta} \ln \left(\frac{\alpha}{t_{i}}\right) M\left(t_{i}, \theta\right)}{1-M\left(t_{i}, \theta\right)}\right], \\
& \widehat{i_{15}}=\frac{1}{n} \sum_{i=1}^{n} \delta_{i}\left[\frac{1-a \gamma\left(\frac{\alpha}{t_{i}}\right)^{\beta}}{a}-\frac{\gamma\left(\frac{\alpha}{t_{i}}\right)^{\beta} M\left(t_{i}, \theta\right)}{1-M\left(t_{i}, \theta\right)}\right]\left[\frac{1-a \gamma\left(\frac{\alpha}{t_{i}}\right)^{\beta}}{\gamma}-\frac{a\left(\frac{\alpha}{t_{i}}\right)^{\beta} M\left(t_{i}, \theta\right)}{1-M\left(t_{i}, \theta\right)}\right] \text {, }
\end{aligned}
$$




$$
\begin{aligned}
& \widehat{i_{22}}=\frac{1}{n} \sum_{i=1}^{n} \delta_{i} \frac{1}{b^{2}}, \\
& \widehat{i_{23}}=\frac{1}{n} \sum_{i=1}^{n} \delta_{i}\left[\frac{1}{b} \frac{1-a \gamma \alpha \beta \alpha^{\beta-1} t_{i}^{-\beta}}{\alpha}-\frac{a \gamma \beta \alpha^{\beta-1} t_{i}^{-\beta} M\left(t_{i}, \theta\right)}{1-M\left(t_{i}, \theta\right)}\right] \text {, } \\
& \widehat{i_{24}}=\frac{1}{n} \sum_{i=1}^{n} \delta_{i}\left[\frac{1}{b} \frac{1-\beta \ln \left(t_{i}\right)-\beta a \gamma\left(\frac{\alpha}{t_{i}}\right)^{\beta} \ln \left(\frac{\alpha}{t_{i}}\right)}{\beta}-\frac{a \gamma\left(\frac{\alpha}{t_{i}}\right)^{\beta} \ln \left(\frac{\alpha}{t_{i}}\right)^{\beta} M\left(t_{i}, \theta\right)}{1-M\left(t_{i}, \theta\right)}\right] \text {, } \\
& \widehat{i_{25}}=\frac{1}{n} \sum_{i=1}^{n} \delta_{i}\left[\frac{1}{b} \frac{1-a \gamma\left(\frac{\alpha}{t_{i}}\right)^{\beta}}{\gamma}-\frac{a\left(\frac{\alpha}{t_{i}}\right) M\left(t_{i}, \theta\right)}{1-M\left(t_{i}, \theta\right)}\right] \\
& \widehat{i_{33}}=\frac{1}{n} \sum_{i=1}^{n} \delta_{i}\left[\frac{1-a \gamma \alpha \beta \alpha^{\beta-1} t_{i}^{-\beta}}{\alpha}-\frac{a \gamma \beta \alpha^{\beta-1} t_{i}^{-\beta} M\left(t_{i}, \theta\right)}{1-M\left(t_{i}, \theta\right)}\right]^{2}, \\
& \widehat{i_{34}}=\frac{1}{n} \sum_{i=1}^{n} \delta_{i}\left[\frac{1-a \gamma \alpha \beta \alpha^{\beta-1} t_{i}^{-\beta}}{\alpha}-\frac{a \gamma \beta \alpha^{\beta-1} t_{i}^{-\beta} M\left(t_{i}, \theta\right)}{1-M\left(t_{i}, \theta\right)}\right] \\
& \times\left[\frac{1-\beta \ln \left(t_{i}\right)-\beta a \gamma\left(\frac{\alpha}{t_{i}}\right)^{\beta} \ln \left(\frac{\alpha}{t_{i}}\right)}{\beta}-\frac{a \gamma\left(\frac{\alpha}{t_{i}}\right)^{\beta} \ln \left(\frac{\alpha}{t_{i}}\right) M\left(t_{i}, \theta\right)}{1-M\left(t_{i}, \theta\right)}\right], \\
& \widehat{i_{35}}=\frac{1}{n} \sum_{i=1}^{n} \delta_{i}\left[\frac{1-\alpha \gamma \alpha \beta \alpha^{\beta-1} t_{i}^{-\beta}}{\alpha}-\frac{a \gamma \beta \alpha^{\beta-1} t_{i}^{-\beta} M\left(t_{i}, \theta\right)}{1-M\left(t_{i}, \theta\right)}\right]\left[\frac{1-\alpha \gamma\left(\frac{\alpha}{t_{i}}\right)^{\beta}}{\gamma}-\frac{a\left(\frac{\alpha}{t_{i}}\right)^{\beta} M\left(t_{i}, \theta\right)}{1-M\left(t_{i}, \theta\right)}\right] \text {, } \\
& \widehat{i_{44}}=\frac{1}{n} \sum_{i=1}^{n} \delta_{i}\left[\frac{1-\beta \ln \left(t_{i}\right)-\beta a \gamma\left(\frac{\alpha}{t_{i}}\right)^{\beta} \ln \left(\frac{\alpha}{t_{i}}\right)}{\beta}-\frac{a \gamma\left(\frac{\alpha}{t_{i}}\right)^{\beta} \ln \left(\frac{\alpha}{t_{i}}\right) M\left(t_{i}, \theta\right)}{1-M\left(t_{i}, \theta\right)}\right]^{2},
\end{aligned}
$$




$$
\begin{aligned}
\widehat{i_{45}}=\frac{1}{n} \sum_{i=1}^{n} \delta_{i}\left[\frac{1-\beta \ln \left(t_{i}\right)-\beta a \gamma\left(\frac{\alpha}{t_{i}}\right)^{\beta} \ln \left(\frac{\alpha}{t_{i}}\right)}{\beta}-\frac{a \gamma\left(\frac{\alpha}{t_{i}}\right)^{\beta} \ln \left(\frac{\alpha}{t_{i}}\right) M\left(t_{i}, \theta\right)}{1-M\left(t_{i}, \theta\right)}\right] \\
\\
\times\left[\frac{1-a \gamma\left(\frac{\alpha}{t_{i}}\right)^{\beta}}{\gamma}-\frac{a\left(\frac{\alpha}{t_{i}}\right)^{\beta} M\left(t_{i}, \theta\right)}{1-M\left(t_{i}, \theta\right)}\right], \\
\widehat{i_{55}}=\frac{1}{n} \sum_{i=1}^{n} \delta_{i}\left[\frac{1-a \gamma\left(\frac{\alpha}{t_{i}}\right)^{\beta}}{\gamma}-\frac{a\left(\frac{\alpha}{t_{i}}\right)^{\beta} M\left(t_{i}, \theta\right)}{1-M\left(t_{i}, \theta\right)}\right]^{2} .
\end{aligned}
$$

